\title{
Investigation of candidate genes for metabolic disorders expressed in liver and pituitary gland by comparing the RNA-seq data of Polish-HF and Polish-Red cattle
}

Dominika Wysocka ${ }^{1}$, Przemysław Sobiech ${ }^{1}$, Magdalena Herudzińska ${ }^{3}$, Mateusz Sachajko ${ }^{2,3}$, Chandra S. Pareek ${ }^{2,3}$

\footnotetext{
${ }^{1}$ Department and Clinic of Internal Diseases, Faculty of Veterinary Medicine, University of Warmia and Mazury in Olsztyn, 10-719 Olsztyn, Poland ${ }^{2}$ Centre for Modern Interdisciplinary Technologies, Nicolaus Copernicus University, Toruń, Poland ${ }^{3}$ Centre of Veterinary Sciences, Inter-university Centre of Veterinary Medicine, Nicolaus Copernicus University, Toruń, Poland
}

Corresponding author:

Prof. dr hab. Chandra S. Pareek

Division of Functional Genomics in Biological and Biomedical Research,

Centre for Modern Interdisciplinary Technologies, Nicolaus Copernicus University,

ul. Wileńska 4, 87-100 Toruń, Poland

E-mail: pareekcs@umk.pl 
Abstract. Background: Metabolic disorder is a major health problem in dairy cattle, particularly to high milk producing dairy cattle. It is worthily emphasized that metabolic diseases have a very complex etiology and pathogenesis, and the impact of these diseases on hepatic and pituitary gland gene expression and organism oxidative balance is not fully described. The presented study was aimed to determine and predict the hepatic and pituitary gland expression of potential candidate genes in context to maintenance of oxidative balance, negative nitrogen balance, as well as ketosis in Polish HF and Polish Red cattle.

Methods: Based on the RNA-seq experimental data, we investigated the candidate genes (SOD1, SOD2, SOD3, GPx2, GPx3, GPx5, GPx6, GPx7, GPx8, BDH1, FN1, ACSL3, HMGCL, HMGCS2, BDH2, ACSL6, ACAT2, IDH3B, ACAT1, HMGCS1, ACSL4, ACSL1, $P C, C P T 1 A, O X C T 1$ and $A C S L 5$ respectively) expressions in liver and pituitary gland tissues of Polish HF and Polish Red cattle. The RNA-seq experimental design comprised of young bulls aged between 6 to 12 months were investigated. For each breed, six liver and six pituitary gland tissues were sequenced using Next-seq 500 illumina platform. The RNA-seq expression data were normalized by the reads per kilobase of exon per million reads mapped (RPKM) method.

Results: By comparing the RNA-seq data of liver and pituitary gland tissues, the investigated candidate genes were highly expressed in the hepatic tissues than to pituitary gland in investigated cattle breeds. However, by comparing the Polish HF and Polish Red cattle breeds, results revealed a similar trend of gene expression profiling of all investigated candidate genes for both metabolic tissues. In case of hepatic gene expression profiling, the SOD1, FN1, HMGCL, HMGCS2, ACAT2, ACAT1, HMGCS1, ACSL1 and ACSL5 were highly expressed (FPKM values of $>40$ ), followed by $S O D 2, G P X 3, I D H 3 B, P C$ and $B D H 2$ as moderately expressed (FPKM values: $>10$ to $<40$ ), and averagely expressed SOD3, GPX5, GPX6, GPX7, GPX2, GPX8, BDH1, ACSL3, ACSL6, ACSL4, CPT1A and OXCT1 respectively, in Polish HF and Polish Red breeds. In case of pituitary gland gene expression profiling, the SOD1 and GPx3 were highly expressed (FPKM values of $>40$ ), followed by SOD2, GPX8, IDH3B, ACAT1, ACSL 4 and PC as moderately expressed (FPKM values: $>10$ to $<40$ ), and averagely expressed SOD3, GPX3,GPX5, GPX6, GPX7, GPX2, BDH1, BDH2, ACSL3, ACSL6, CPT1A, OXCT1, FN1, HMGCL, HMGCS2, ACAT2, ACAT1, HMGCS1, ACSL1 and ACSL5 respectively, in Polish HF and Polish Red breeds.

Conclusions: Based on this presented results on hepatic and pituitary gland gene expression, a further research plan is an essential pre-requisite to validate the identified candidate genes. Study indicated the understanding the genetic factors that predispose metabolic disorders in cattle would benefit the dairy industry as a whole by providing producers, breeding services, and veterinarians a tool to forecast a cow's susceptibility to metabolic disorders.

Keywords: RNA-seq; liver, pituitary gland; cattle; breeds; ketosis; SOD; GPx; antioxidants; bioinformatics. 


\section{Introduction}

Metabolic diseases and disturbances of oxidative balance (oxidative stress) are constantly current threats to the health and welfare of dairy cows as well as the profitability of dairy farms and dairy industry all around the world [1-2]. The breeding selection and the increasing intensification of milk production made the high-yielding dairy cow an animal with a huge metabolic burden and susceptibility to metabolic diseases, such as ketosis [3-4]. High-yielding animals due to the very high rate of number of metabolic processes are also more susceptible to oxidative balance disturbances [5]. Both metabolic diseases and negative effects of oxidative stress contribute to shortening the life of animals or the need of culling them from the herd, and thus generate enormous economic losses in the dairy industry [6-7]. In recent year increased interest in ketosis and genetic studies have been observed in several manuscripts published in most reputable journals [8-13]. Furthermore there is increased interest in breed differences in transcriptome of Bos Taurus [14]. Ketosis in dairy cow is a multifactorial disease that is the result of a maladaptive response to negative energy balance (NEB) in early lactation. While essentially all cows undergo NEB after calving, not all cows develop ketosis. Recent study observed variable metabolic loads and differential gene expression in the liver of cows that had been pre-selected for high milk fat; despite being selected for high F:P, an indicator of ketosis, the susceptibility to ketosis was different for cows within this group [15]. Moreover, some other studies suggest that altered metabolic regulation that contributes to ketosis differs at the cow level [16-20]. Trait-association analyses that have been conducted for metabolic traits - energy balance, F:P, GPC:PC, blood $\mathrm{BHB}, \mathrm{BCS}$, milk metabolites, EBV - have identified regions of the genome, candidate genes and probable quantitative trait nucleotides (QTN) that explain some of the gene expression variation between cows [21-27]. However, further exploration of candidate genes for ketosis could contribute to our understanding of the genetic architecture of this trait. The construction of a custom designed array of candidate genes specific to the 
trait of interest would allow for the investigation of many key genes simultaneously, as well as identify probable QTN with biological significance. In the paper, we investigated the gene expression variations of the GPx and SOD gene families involved in the maintenance of oxidative balance, as well as, gene expression variations among recently identified candidate genes for ketosis using the RNA-seq data of liver and pituitary gland tissues.

\section{Materials and methods}

Animals: The RNA-seq experimental design comprised of young bulls aged between 6 to 12 months were investigated. For each breed, six liver and pituitary gland tissues were sequenced using Next-seq 500 illumina platform (Figure 1). All procedures involving animals were performed in accordance with the guiding principles for the care and use of research animals. The investigated liver and pituitary gland tissues from PolishHF and Polish Red cattle were approved by the local ethics commission of IGAB, PAS, Jastrzębiec, Poland (permission No. 3/2005).

RNA-seq laboratory work: Isolation of total RNA from liver and pituitary gland tissues were prepared from 50-60 mg of frozen tissues using the guanidinium thiocyanate method [28] (TRIzol reagent: Invitrogen, Carlsbad, CA, USA). All RNA samples were evaluated with the Agilent BioAnalyzer using the Nano RNA Kit with RNA Integrity Number $($ RIN) $>7.0$. For the library preparation $5 \mu$ g were total RNA and two biological replicates were used for each investigated tissue. The mRNA isolation was carried out by using the Dynabeads mRNA Direct" kit (Thermo Fisher), and followed by dUTP directional mRNA libraries preparation, according to the NEBNext Ultra Directional RNA library preparation Kit for Illumina (New England Bio Labs). The cDNA fragments were end-repaired, A-tailed, and ligated to the TruSeq y-tail single indexes from Illumina TruSeq DNA kit (Figure 1). Indexed libraries were cut with USER enzyme, and PCR amplified for 12 cycles. To achieve the highest quality data on Illumina sequencing platforms, optimum cluster 


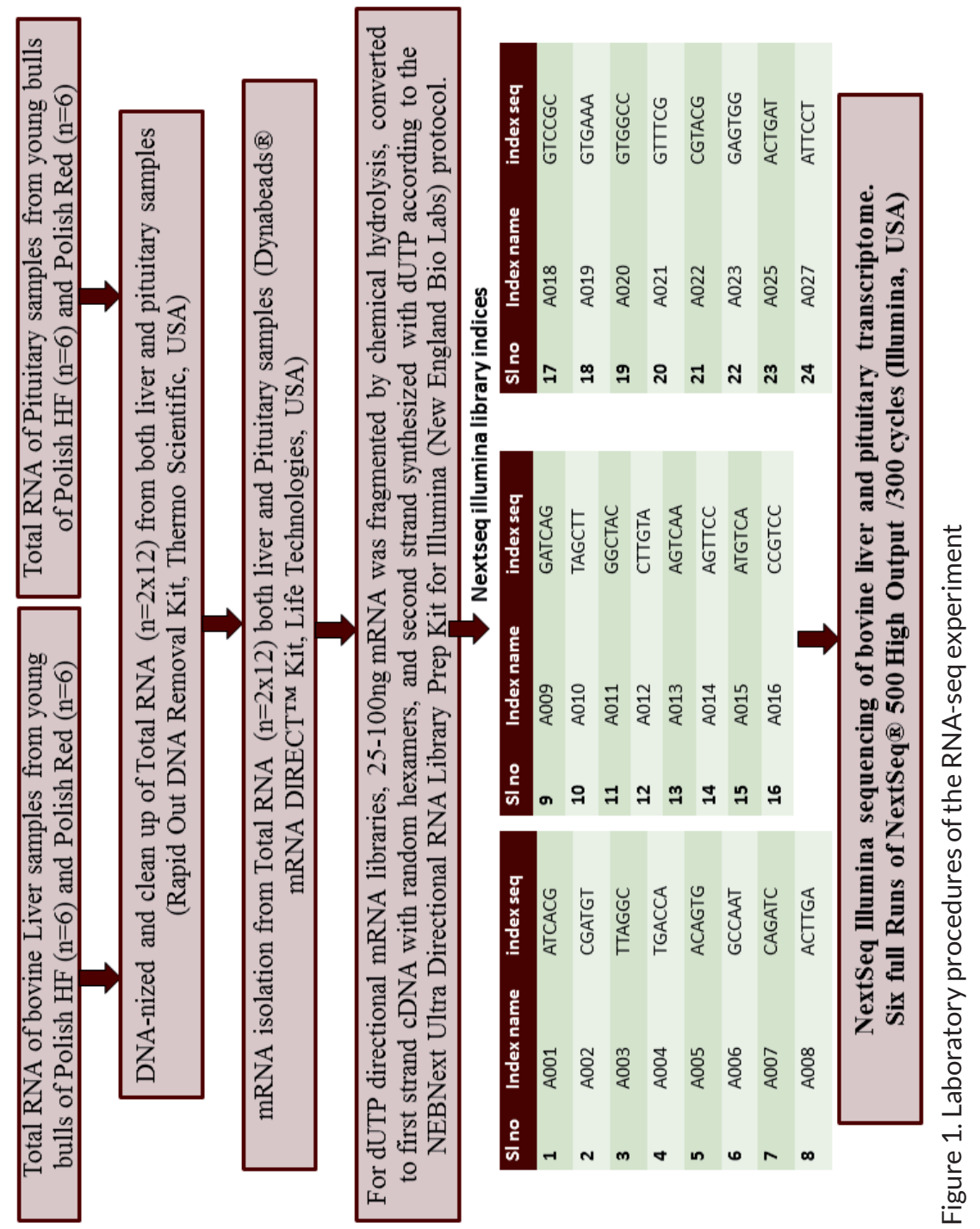


deposition was made by quantitation of libraries using qPCR according to the Illumina Sequencing Library qPCR Quantification Guide (Kapa Biosciences). Finally, 156x156 bp paired-end sequence reads were generated using the Illumina NextSeq 500 platform High Output/300 cycle kits from Illumina.

Bioinformatics analysis: For all investigated liver and pituitary gland tissues $(\mathrm{n}=12)$, the minimum overlap length was set to 10 and error rate was set to 0.05 at cutadapt software [29]. After cutting adaptor, the low quality bases were trimmed from 3'- end. The processed short paired end reads were aligned, or mapped to the reference genome Ensembl75_ UMD3-1.1 plus the Chromosome Y from Btau_4.6.1 assembly, by using BWA version 0.7.5-r404 [30]. The HT-Seq framework, version 0.5.3p9, was used to count the aligned reads in genes using the STAR BWA tools [31]. Finally, the RNA-seq expression data were normalized by the reads per kilobase of exon per million reads mapped (RPKM) method [32] to identify the gene expression variations of the candidate genes of ketosis in bovine liver and pituitary gland transcriptome.

\section{Results and discussion}

Based on the RNA-seq data, we have screened and investigated the known CGs for liver and pituitary gland transcriptome as presented in Table 1. By comparing the RNA-seq data of two metabolic tissues, study revealed that the investigated candidate genes were highly expressed in the hepatic tissues than to pituitary gland in cattle breeds (Table 2 and Table 3 ). However, by comparing the Polish HF and Polish Red cattle, results revealed a similar trend of gene expression profiling of all investigated candidate genes for both metabolic tissues (Table 2 and Table 3). Furthermore based on the obtained results, we have categorized gene expression profiling as: highly, moderately and averagely expressed candidate genes. In case of hepatic gene expression profiling, the SOD1, FN1, HMGCL, HMGCS2, ACAT2, ACAT1, HMGCS1, ACSL1 and ACSL5 were highly expressed (FPKM values of $>40$ ), followed by SOD2, GPX3, IDH3B, PC and BDH2 


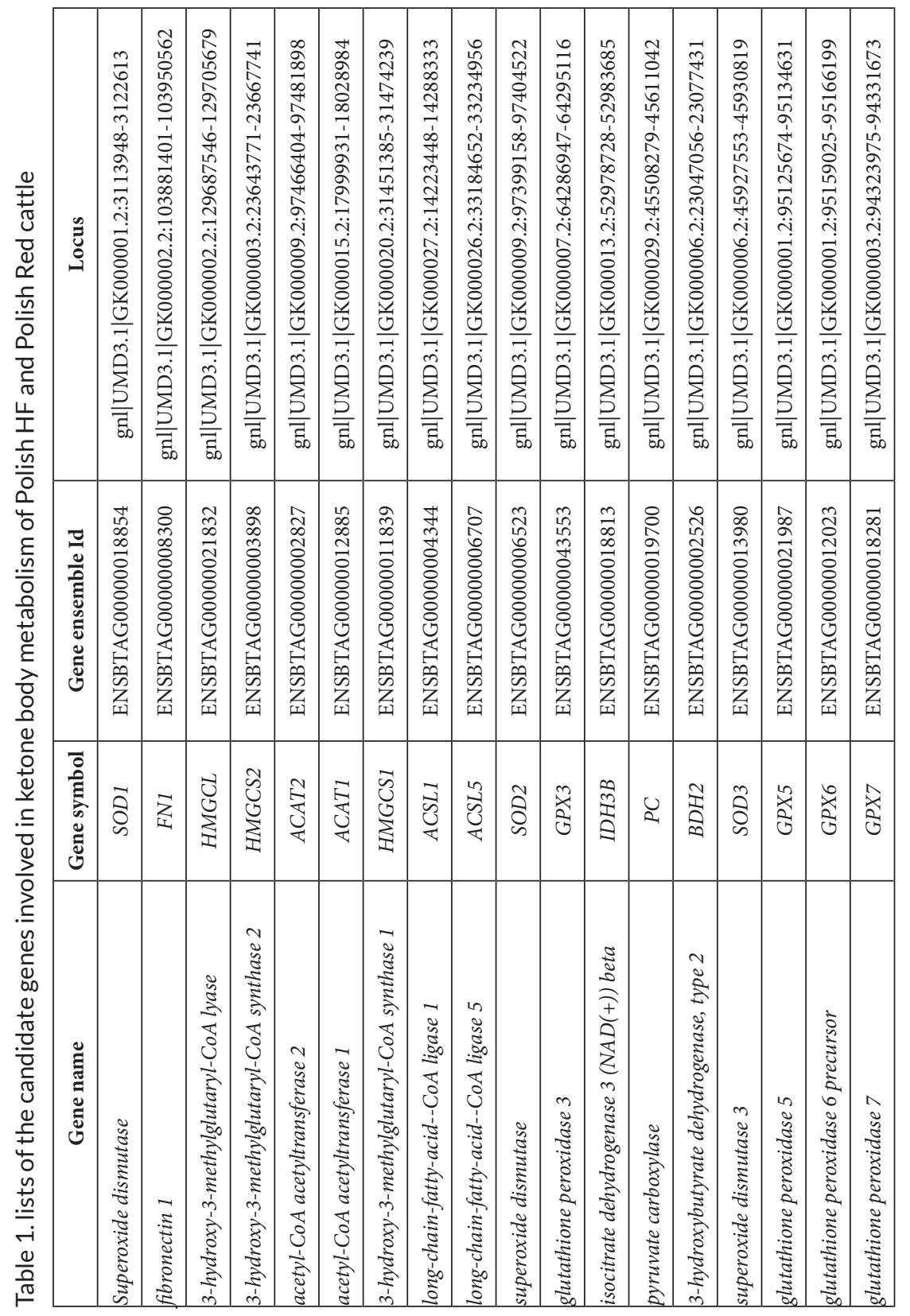



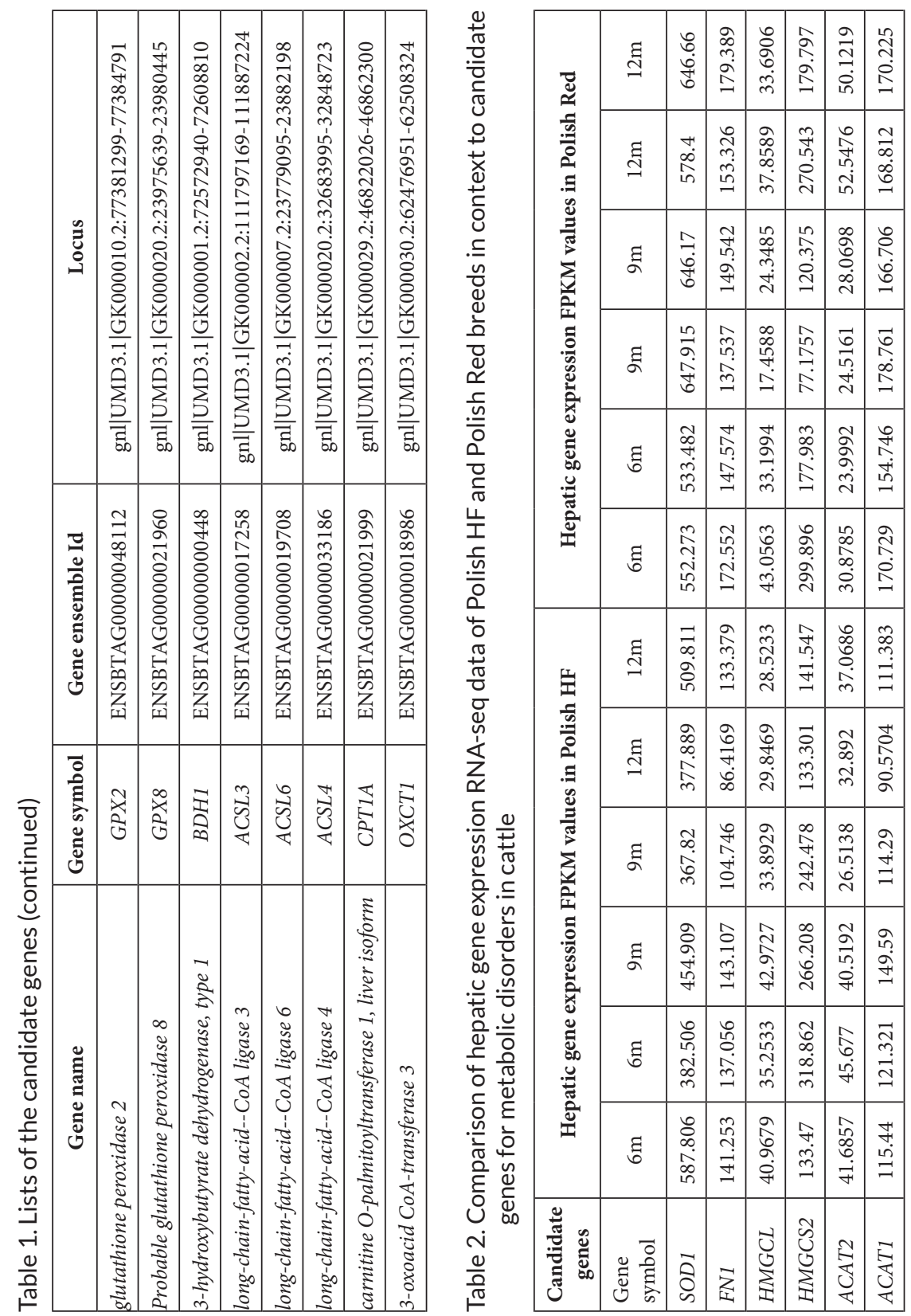


\begin{tabular}{|c|c|c|c|c|c|c|c|c|c|c|c|c|c|c|c|c|c|c|c|c|}
\hline $\overrightarrow{\mathscr{Z}}$ & 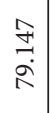 & $\begin{array}{l}\infty \\
\stackrel{\infty}{\sim} \\
\stackrel{I}{I}\end{array}$ & 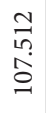 & 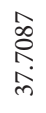 & ָิ & $\begin{array}{l}\vec{n} \\
\hat{n} \\
\stackrel{n}{-}\end{array}$ & $\begin{array}{l}\vec{\sigma} \\
\vec{\sigma} \\
\dot{\rho} \\
\dot{m}\end{array}$ & $\begin{array}{l}\vec{\sigma} \\
\dot{0} \\
\dot{+} \\
\vec{\nu}\end{array}$ & 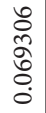 & \begin{tabular}{|l|} 
\\
0 \\
0 \\
0 \\
0 \\
0
\end{tabular} & $\begin{array}{l}8 \\
8 \\
0 \\
0 \\
0\end{array}$ & $\begin{array}{l}n \\
\hat{s} \\
\delta \\
i\end{array}$ & $\begin{array}{l}\stackrel{\infty}{\circ} \\
\infty \\
\stackrel{+}{\mathbb{2}} \\
\stackrel{0}{0}\end{array}$ & 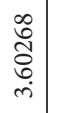 & $\begin{array}{l}8 \\
\vdots \\
\vdots \\
\vdots \\
0\end{array}$ & 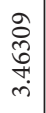 & 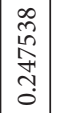 & 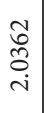 & $\begin{array}{l}m \\
\infty \\
\infty \\
\stackrel{2}{1} \\
\end{array}$ & $\begin{array}{l}\overrightarrow{0} \\
\stackrel{a}{\hat{n}} \\
\text { in }\end{array}$ \\
\hline $\begin{array}{l}5 \\
\\
0\end{array}$ & 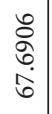 & 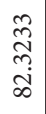 & $\exists$ & 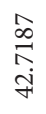 & $\frac{6}{4}$ & $\begin{array}{l}\hat{0} \\
\stackrel{2}{0} \\
\infty \\
\text { Non }\end{array}$ & $\begin{array}{l}\text { शे } \\
\hat{n} \\
\stackrel{\text { ते }}{2}\end{array}$ & 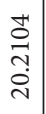 & \begin{tabular}{c}
$\vec{S}$ \\
\multirow{J}{+}{} \\
-
\end{tabular} & \begin{tabular}{|l|}
8 \\
0 \\
0 \\
0 \\
0 \\
0
\end{tabular} & $\begin{array}{l}0 \\
8 \\
0 \\
0 \\
0\end{array}$ & 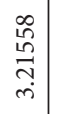 & $\begin{array}{l}\hat{0} \\
\infty \\
\hat{n} \\
\vdots \\
0\end{array}$ & 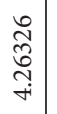 & $\begin{array}{l}8 \\
\vdots \\
\vdots \\
\vdots\end{array}$ & 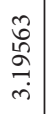 & $\begin{array}{l}\vec{n} \\
\hat{n} \\
n \\
n \\
0 \\
0\end{array}$ & 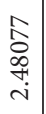 & 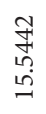 & $\begin{array}{l}\infty \\
\infty \\
\infty \\
0 \\
0 \\
\dot{m}\end{array}$ \\
\hline 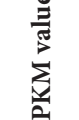 & $\begin{array}{l}\stackrel{0}{2} \\
\infty \\
\infty \\
\dot{0}\end{array}$ & $\begin{array}{l}\infty \\
N \\
\hat{N} \\
\infty \\
\infty \\
+\end{array}$ & $\stackrel{2}{2}$ & 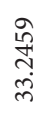 & $\underset{\stackrel{3}{\sim}}{\sim}$ & $\begin{array}{l}\vec{n} \\
\infty \\
\stackrel{n}{0} \\
\end{array}$ & $\begin{array}{l}\tilde{O} \\
\infty \\
\infty \\
i n\end{array}$ & $\begin{array}{l}\stackrel{\Omega}{\cong} \\
= \\
= \\
=\end{array}$ & $\begin{array}{l}\vec{\infty} \\
\stackrel{+}{+} \\
\stackrel{+}{0} \\
0\end{array}$ & \begin{tabular}{|l|}
8 \\
0 \\
0 \\
0 \\
0 \\
0
\end{tabular} & $\begin{array}{l}8 \\
\vdots \\
\vdots \\
0 \\
0\end{array}$ & 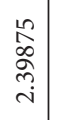 & 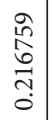 & $\begin{array}{l}\stackrel{0}{=} \\
\stackrel{n}{\sim} \\
\stackrel{\infty}{-}\end{array}$ & $\begin{array}{l}8 \\
\vdots \\
\vdots \\
\vdots\end{array}$ & 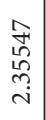 & 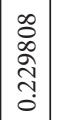 & $\begin{array}{l}\underset{\sim}{\sim} \\
\underset{\infty}{0} \\
\stackrel{-}{-}\end{array}$ & $\begin{array}{l}\text { Oे } \\
\text { ị } \\
=\end{array}$ & $\begin{array}{l}\text { } \\
\stackrel{\sim}{\hat{~}} \\
\text { ஸे }\end{array}$ \\
\hline 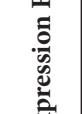 & $\begin{array}{l}\underset{\Xi}{\Xi} \\
\underset{\sigma}{0}\end{array}$ & $\begin{array}{l}\overrightarrow{\widehat{े}} \\
\text { } \\
\text { ेे }\end{array}$ & $\approx$ & $\begin{array}{l}\hat{\tilde{y}} \\
\infty \\
\vec{\eta}\end{array}$ & ธิ & 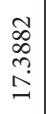 & $\begin{array}{l}\vec{\sigma} \\
\stackrel{+}{+} \\
\stackrel{j}{j}\end{array}$ & 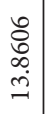 & 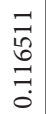 & \begin{tabular}{|l|}
8 \\
0 \\
0 \\
0 \\
0 \\
0
\end{tabular} & $\begin{array}{l}0 \\
0 \\
0 \\
0 \\
0 \\
0\end{array}$ & 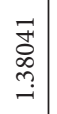 & $\begin{array}{l}0 \\
\vdots \\
\vdots \\
0 \\
0\end{array}$ & 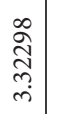 & $\begin{array}{l}8 \\
8 \\
0 \\
0 \\
0\end{array}$ & 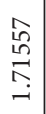 & $\left|\begin{array}{l}0 \\
\stackrel{2}{a} \\
2 \\
\stackrel{1}{0}\end{array}\right|$ & 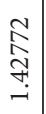 & 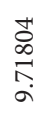 & $\begin{array}{l}\overrightarrow{\widehat{\sigma}} \\
\hat{o} \\
\dot{n}\end{array}$ \\
\hline 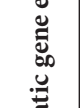 & 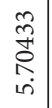 & 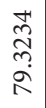 & $\stackrel{\infty}{\mathbb{N}}$ & 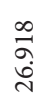 & $\begin{array}{l}\stackrel{m}{\exists} \\
\stackrel{\Xi}{I}\end{array}$ & 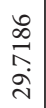 & 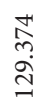 & $\begin{array}{l}\text { I } \\
\vec{n} \\
\stackrel{\lambda}{n}\end{array}$ & $\begin{array}{l}\stackrel{2}{\infty} \\
\infty \\
\stackrel{\sim}{\rightarrow}\end{array}$ & $\mid \begin{array}{l}8 \\
0 \\
0 \\
0 \\
0 \\
0\end{array}$ & $\begin{array}{l}0 \\
0 \\
0 \\
0 \\
0\end{array}$ & \begin{tabular}{l|}
$\infty$ \\
$\infty$ \\
$\dot{n}$ \\
$\hat{n}$ \\
$\dot{m}$
\end{tabular} & $\begin{array}{l}0 \\
\vdots \\
0 \\
0 \\
0\end{array}$ & 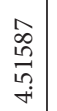 & 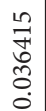 & 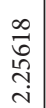 & 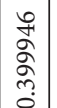 & 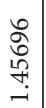 & 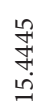 & 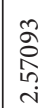 \\
\hline$=$ & 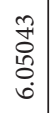 & 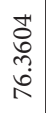 & స్ & $\stackrel{\infty}{\sigma}$ & Iิ & $\begin{array}{l}\hat{N} \\
\hat{2} \\
\sigma \\
\text { in } \\
m\end{array}$ & 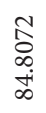 & $\begin{array}{l}\mathscr{m} \\
\underset{g}{q} \\
\end{array}$ & $\begin{array}{l}n \\
0 \\
\tilde{0} \\
0 \\
0\end{array}$ & \begin{tabular}{|l|}
8 \\
0 \\
0 \\
0 \\
0 \\
0
\end{tabular} & $\begin{array}{l}8 \\
8 \\
0 \\
0 \\
0\end{array}$ & $\mid \begin{array}{l}n \\
\stackrel{0}{0} \\
\sigma \\
\sigma \\
+ \\
+\end{array}$ & $\begin{array}{c}\infty \\
\stackrel{1}{2} \\
\tilde{0} \\
0\end{array}$ & 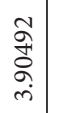 & $\begin{array}{l}8 \\
8 \\
0 \\
0 \\
0\end{array}$ & 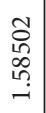 & 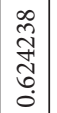 & 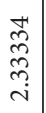 & $\begin{array}{l}\infty \\
\infty \\
\infty \\
\infty \\
= \\
=\end{array}$ & $\begin{array}{l}\text { Lे } \\
\hat{\sigma} \\
\dot{\sigma}\end{array}$ \\
\hline & 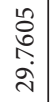 & 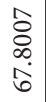 & 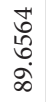 & $\begin{array}{l}\hat{N} \\
\hat{\sigma} \\
\hat{i n} \\
0\end{array}$ & $\stackrel{n}{n}$ & 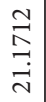 & 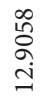 & ถู่ิ & 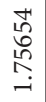 & \begin{tabular}{|l|}
8 \\
0 \\
0 \\
0 \\
0 \\
0
\end{tabular} & $\begin{array}{l}8 \\
8 \\
0 \\
0 \\
0\end{array}$ & 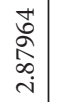 & $\begin{array}{l}8 \\
\vdots \\
0 \\
0 \\
0\end{array}$ & \begin{tabular}{l}
$\infty$ \\
$\stackrel{2}{\$}$ \\
\multirow{+}{0}{} \\
in
\end{tabular} & $\begin{array}{l}8 \\
\vdots \\
\vdots \\
\vdots \\
0\end{array}$ & $\begin{array}{l}\stackrel{-}{\infty} \\
\infty \\
\stackrel{-}{-}\end{array}$ & $\mid$\begin{tabular}{c|}
$n$ \\
0 \\
2 \\
0 \\
1 \\
0 \\
0
\end{tabular} & \begin{tabular}{l}
$\infty$ \\
\multirow{0}{0}{} \\
$\overrightarrow{-}$ \\
\end{tabular} & $\begin{array}{l}2 n \\
\hat{n} \\
\hat{n} \\
\sigma \\
i n\end{array}$ & 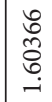 \\
\hline $\begin{array}{l}\frac{5}{0} \\
. \\
. \Xi\end{array}$ & 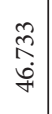 & $\begin{array}{l}\mathcal{N} \\
\hat{N} \\
\infty \\
\infty \\
\infty\end{array}$ & ঙ & $\overrightarrow{\widehat{\grave{j}}}$ & స్ర & \begin{tabular}{l}
$\infty$ \\
\multirow{2}{0}{} \\
$\stackrel{2}{+}$
\end{tabular} & $\begin{array}{l}\hat{\kappa} \\
\stackrel{\text { సે }}{=}\end{array}$ & 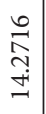 & $\begin{array}{l}\mathbb{N} \\
\stackrel{0}{0} \\
\stackrel{0}{0} \\
0\end{array}$ & $\begin{array}{l}8 \\
0 \\
0 \\
0 \\
0 \\
0\end{array}$ & $\begin{array}{l}8 \\
8 \\
0 \\
0 \\
0\end{array}$ & 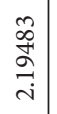 & 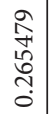 & 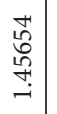 & $\begin{array}{l}8 \\
8 \\
\vdots \\
0\end{array}$ & $\begin{array}{l}\stackrel{+}{N} \\
\stackrel{\hat{D}}{0} \\
\stackrel{-}{-}\end{array}$ & 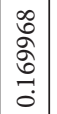 & $\begin{array}{l}\infty \\
0 \\
0 \\
\infty \\
n \\
n\end{array}$ & $\begin{array}{l}\stackrel{N}{1} \\
\stackrel{0}{0} \\
\dot{0}\end{array}$ & 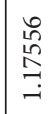 \\
\hline$\sum_{3}^{\pi}$ & 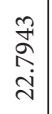 & 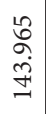 & 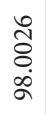 & $\begin{array}{l}\infty \\
\mathbb{H} \\
\text { ț }\end{array}$ & $\begin{array}{l}\text { f } \\
\text { a }\end{array}$ & 눙 & $\begin{array}{l}\infty \\
\infty \\
\stackrel{0}{0} \\
\infty\end{array}$ & $\begin{array}{l}\vec{\Delta} \\
\hat{0} \\
\stackrel{-}{a}\end{array}$ & 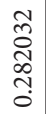 & $\begin{array}{l}8 \\
8 \\
0 \\
0 \\
0\end{array}$ & $\begin{array}{l}8 \\
8 \\
8 \\
0 \\
0\end{array}$ & $\begin{array}{l}\hat{\sigma} \\
\vec{m} \\
\dot{m}\end{array}$ & $\begin{array}{l}0 \\
\stackrel{0}{0} \\
\$ \\
\stackrel{-}{\circ} \\
-\end{array}$ & 命 & $\begin{array}{l}\stackrel{+}{N} \\
\stackrel{+}{+} \\
\hat{O} \\
\stackrel{0}{0}\end{array}$ & 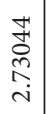 & 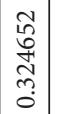 & $\begin{array}{l}\stackrel{n}{a} \\
\underset{N}{N} \\
\infty \\
\infty \\
0\end{array}$ & $\begin{array}{l}\Lambda \\
\infty \\
+\infty \\
\infty \\
+ \\
+\end{array}$ & $\begin{array}{l}\text { న̆ } \\
\text { ते } \\
\text { ते }\end{array}$ \\
\hline 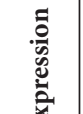 & $\begin{array}{l}\widetilde{\Omega} \\
\widehat{\hat{L}} \\
\infty \\
-\end{array}$ & 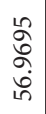 & ร & $\begin{array}{l}\stackrel{2}{2} \\
\stackrel{\sim}{\infty}\end{array}$ & $\vec{\partial}$ & $\hat{\sigma}$ & $\begin{array}{l}\vec{\infty} \\
\tilde{n} \\
0 \\
\tilde{n}\end{array}$ & 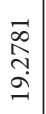 & 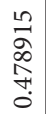 & $\begin{array}{l}0 \\
8 \\
0 \\
0 \\
0 \\
0\end{array} \mid$ & $\begin{array}{l}0 \\
0 \\
0 \\
\vdots \\
0\end{array}$ & $\begin{array}{l}\text { क } \\
\text { Oे }\end{array}$ & $\begin{array}{l}\stackrel{0}{a} \\
\dot{0} \\
\stackrel{0}{0} \\
\stackrel{0}{0}\end{array}$ & 烯 & $\begin{array}{l}\text { ஸे } \\
0 \\
0 \\
0 \\
0 \\
0\end{array}$ & $\begin{array}{l}\underset{\mathrm{J}}{\mathrm{J}} \\
\underset{\mathrm{J}}{\mathrm{N}}\end{array}$ & \begin{tabular}{|c|}
$\infty$ \\
0 \\
0 \\
0 \\
0 \\
$1+1$ \\
0 \\
0
\end{tabular} & $\begin{array}{c}\stackrel{a}{\mathrm{~b}} \\
\stackrel{m}{\circ} \\
\stackrel{-}{-}\end{array}$ & \begin{tabular}{l}
\multirow{2}{\circ}{} \\
$\stackrel{0}{0}$ \\
\end{tabular} & 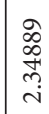 \\
\hline 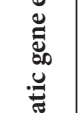 & $\begin{array}{l}\stackrel{\partial}{0} \\
\stackrel{+}{-1} \\
\dot{n}\end{array}$ & 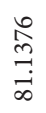 & $\underline{b}$ & $\stackrel{\infty}{\underset{f}{f}}$ & t艹 & $\overrightarrow{0}$ & 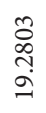 & 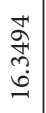 & $\begin{array}{l}\stackrel{0}{2} \\
\stackrel{2}{0} \\
\infty \\
\stackrel{0}{0}\end{array}$ & \begin{tabular}{|l|}
0 \\
0 \\
0 \\
0 \\
0
\end{tabular} & $\begin{array}{l}8 \\
8 \\
8 \\
0 \\
0\end{array}$ & $n$ & $\begin{array}{l}8 \\
\vdots \\
\vdots \\
\vdots \\
\circ\end{array}$ & $\stackrel{\infty}{\wedge}$ & 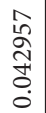 & $\underset{\stackrel{N}{\sim}}{\stackrel{\sim}{\sim}}$ & $\left|\begin{array}{l}10 \\
0 \\
0 \\
0 \\
0 \\
0 \\
0\end{array}\right|$ & \begin{tabular}{l} 
IV \\
$\infty$ \\
$\infty$ \\
$\stackrel{0}{0}$ \\
\multirow{+}{0}{} \\
0
\end{tabular} & $\begin{array}{l}\text { से } \\
\text { ळे } \\
\text { बे }\end{array}$ & 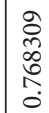 \\
\hline & 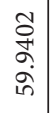 & $\begin{array}{l}\hat{\sigma} \\
\stackrel{0}{0} \\
\hat{\sigma}\end{array}$ & 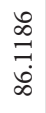 & $\hat{\delta}$ & مิ & $\overrightarrow{\widetilde{N}}$ & $\begin{array}{l}\infty \\
\stackrel{\alpha}{\sigma} \\
\text { in }\end{array}$ & 岁 & 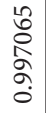 & $\begin{array}{l}0 \\
0 \\
0 \\
0 \\
0 \\
0\end{array}$ & $\begin{array}{l}8 \\
8 \\
0 \\
0 \\
0\end{array}$ & $n$ & $\overrightarrow{\vec{F}}$ & 우 & 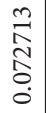 & $\begin{array}{l}\stackrel{+}{ث} \\
\stackrel{1}{0} \\
\infty \\
0\end{array}$ & $\left|\begin{array}{c}0 \\
0 \\
0 \\
\infty \\
\stackrel{1}{1} \\
0\end{array}\right|$ & $\begin{array}{l}\vec{F} \\
\infty \\
m \\
\stackrel{0}{0} \\
\dot{0}\end{array}$ & $\begin{array}{l}\text { Na } \\
\stackrel{\alpha}{\infty} \\
\end{array}$ & 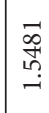 \\
\hline 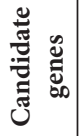 & 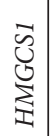 & స్త్ర & & & & $\stackrel{0}{0}$ & 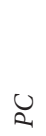 & $\mathbb{I}$ & Оి & $\frac{1}{2}$ & 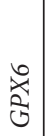 & 㐫 & त्ત્心 & 心 & $\underset{\infty}{\mathbb{I}}$ & 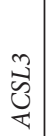 & 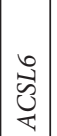 & 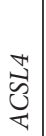 & $\underset{\mathbb{E}}{\mathbb{S}}$ & 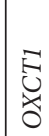 \\
\hline
\end{tabular}




\begin{tabular}{|c|c|c|c|c|c|c|c|c|c|c|c|c|c|}
\hline \multirow{6}{*}{ 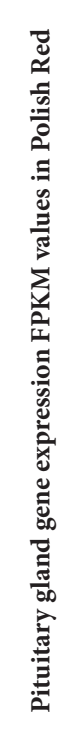 } & $\underset{\text { త్ }}{ }$ & 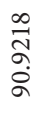 & $\begin{array}{l}\infty \\
\sigma \\
\infty \\
0 \\
0 \\
0\end{array}$ & $\stackrel{\infty}{\stackrel{\infty}{\leftrightarrows}}$ & $\begin{array}{l}\text { ते } \\
\text { ஸे } \\
\stackrel{\sigma}{0}\end{array}$ & $\begin{array}{l}\hat{\Omega} \\
\hat{\tilde{丶}}\end{array}$ & 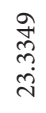 & 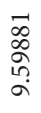 & $\begin{array}{l}\stackrel{H}{d} \\
\text { ָู }\end{array}$ & $\begin{array}{l}\stackrel{10}{0} \\
\infty \\
8 \\
\\
-\end{array}$ & $\begin{array}{l}\text { f } \\
\text { I } \\
\stackrel{5}{0} \\
\stackrel{0}{0}\end{array}$ & 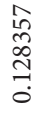 & $\begin{array}{l}\stackrel{+}{N} \\
\stackrel{N}{N} \\
\text { in }\end{array}$ \\
\hline & $\underset{\text { ป }}{\text { I }}$ & $\begin{array}{l}\stackrel{શ}{\hat{~}} \\
\stackrel{\overbrace{}}{=} \\
=\end{array}$ & 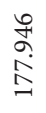 & \begin{tabular}{l}
0 \\
\multirow{+}{+}{} \\
\multirow{2}{*}{}
\end{tabular} & $\begin{array}{l}\hat{A} \\
\hat{\sigma} \\
\stackrel{+}{-}\end{array}$ & $\begin{array}{l}\stackrel{n}{N} \\
\stackrel{\lambda}{ה}\end{array}$ & 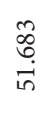 & $\begin{array}{l}\text { Lి } \\
\text { m. } \\
\text { ? }\end{array}$ & $\begin{array}{l}\vec{F} \\
\infty \\
\\
\sigma\end{array}$ & $\begin{array}{l}\text { H } \\
\text { S } \\
\stackrel{5}{4} \\
\text { i }\end{array}$ & \begin{tabular}{l}
$\hat{\delta}$ \\
\multirow{0}{0}{} \\
-1 \\
0
\end{tabular} & 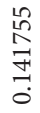 & $\begin{array}{l}\overrightarrow{5} \\
\infty \\
\infty \\
+ \\
+ \\
+\end{array}$ \\
\hline & ฐี & $\begin{array}{l}\infty \\
\infty \\
0 \\
0 \\
0 \\
0\end{array}$ & 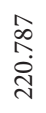 & 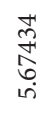 & $\begin{array}{l}0 \\
\hat{0} \\
\swarrow n \\
\hat{n} \\
0\end{array}$ & $\begin{array}{l}\stackrel{m}{0} \\
\stackrel{\infty}{N} \\
\stackrel{n}{n}\end{array}$ & $\begin{array}{l}\stackrel{2}{0} \\
\stackrel{0}{0} \\
\ddot{\sim}\end{array}$ & 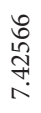 & $\begin{array}{l}\stackrel{0}{\sharp} \\
\stackrel{\mathbb{N}}{N} \\
\text { in }\end{array}$ & 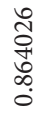 & $\begin{array}{l}8 \\
8 \\
8 \\
0 \\
0\end{array}$ & 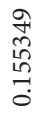 & 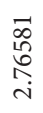 \\
\hline & ઘू & $\begin{array}{l}\tilde{N} \\
\infty \\
\infty \\
=\end{array}$ & 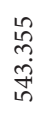 & 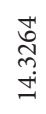 & $\begin{array}{l}\stackrel{N}{ล} \\
\text { å }\end{array}$ & $\begin{array}{l}\stackrel{2}{+} \\
\stackrel{\sim}{N}\end{array}$ & $\begin{array}{l}\overrightarrow{\widetilde{N}} \\
\stackrel{\sim}{n}\end{array}$ & $\begin{array}{l}\text { Oे } \\
\stackrel{\leftrightarrow}{+} \\
\stackrel{9}{-}\end{array}$ & $\begin{array}{l}\overrightarrow{0} \\
\text { ర్ } \\
\stackrel{N}{0}\end{array}$ & \begin{tabular}{l}
\multirow{H}{H}{} \\
$\infty$ \\
0 \\
0 \\
0 \\
0 \\
0
\end{tabular} & 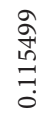 & 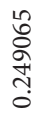 & $\begin{array}{l}0 \\
\stackrel{+}{1} \\
0 \\
n \\
0\end{array}$ \\
\hline & పี & 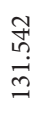 & $\begin{array}{l}\vec{\infty} \\
\vdots \\
\hat{0} \\
\hat{~}\end{array}$ & 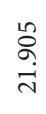 & 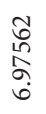 & $\begin{array}{l}\vec{\infty} \\
\vec{m}\end{array}$ & $\begin{array}{l}\vec{H} \\
\widehat{\sigma} \\
\text { } \\
+\end{array}$ & $\begin{array}{l}\vec{\nabla} \\
\infty \\
\stackrel{0}{-}\end{array}$ & $\begin{array}{l}10 \\
0 \\
0 \\
0 \\
0\end{array}$ & 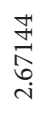 & 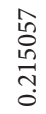 & $\begin{array}{l}\stackrel{n}{N} \\
\stackrel{n}{=} \\
0\end{array}$ & 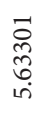 \\
\hline & ีี & $\begin{array}{l}\stackrel{H}{N} \\
\stackrel{\leftrightarrow}{\cong} \\
\end{array}$ & 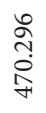 & $\begin{array}{l}\hat{\sigma} \\
\hat{\circ} \\
\alpha \\
\sigma\end{array}$ & $\begin{array}{l}\stackrel{2}{0} \\
\text { ô } \\
= \\
=\end{array}$ & $\begin{array}{l}\mathbb{N} \\
\hat{L} \\
+ \\
+ \\
+\end{array}$ & $\begin{array}{l}\hat{n} \\
\hat{n} \\
\stackrel{n}{n} \\
\hat{m}\end{array}$ & $\begin{array}{l}\stackrel{2}{2} \\
\stackrel{n}{2} \\
\stackrel{n}{=}\end{array}$ & & 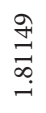 & 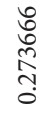 & 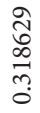 & $\begin{array}{l}+1 \\
\frac{1}{n} \\
\hat{n} \\
+ \\
+1\end{array}$ \\
\hline \multirow{6}{*}{ 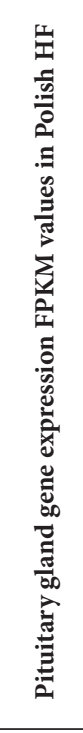 } & $\underset{\beth}{\Xi}$ & 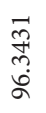 & $\underset{\stackrel{0}{N}}{\stackrel{N}{N}}$ & $\begin{array}{l}\text { } \\
\text { बे } \\
\text { iे }\end{array}$ & \begin{tabular}{l}
$\stackrel{0}{7}$ \\
$\underset{\infty}{\infty}$ \\
\multirow{\sigma}{*}{} \\
$\sigma$
\end{tabular} & $\begin{array}{l}\stackrel{1}{N} \\
\stackrel{\alpha}{\alpha} \\
\stackrel{\infty}{\sim}\end{array}$ & 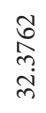 & $\begin{array}{l}\stackrel{0}{\infty} \\
\stackrel{2}{N} \\
\end{array}$ & & 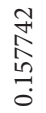 & 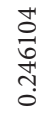 & $\begin{array}{l}8 \\
\vdots \\
\vdots\end{array}$ & 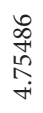 \\
\hline & త్త్ & $\begin{array}{l}\stackrel{0}{i n} \\
\tilde{\tilde{c}} \\
=\end{array}$ & 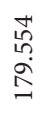 & 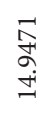 & $\begin{array}{l}\infty \\
\stackrel{\tilde{\Omega}}{0} \\
\stackrel{\Xi}{=}\end{array}$ & $\begin{array}{l}\vec{\sigma} \\
\infty \\
\dot{\sim}\end{array}$ & $\begin{array}{l}\stackrel{a}{\vec{f}} \\
\stackrel{+}{0} \\
\stackrel{\sim}{\sim}\end{array}$ & $\begin{array}{l}\stackrel{n}{\alpha} \\
\stackrel{2}{2} \\
\stackrel{2}{a}\end{array}$ & $\begin{array}{l}\text { Hू } \\
\text { ర్ర } \\
\text { oे }\end{array}$ & 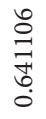 & $\begin{array}{l}\hat{O} \\
\text { Oे } \\
\stackrel{0}{f} \\
\stackrel{0}{0}\end{array}$ & 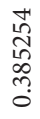 & 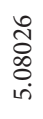 \\
\hline & ઘี & $\begin{array}{l}\stackrel{\hat{~}}{\hat{+}} \\
\stackrel{+}{\infty}\end{array}$ & 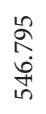 & 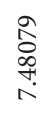 & 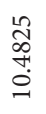 & 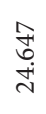 & $\begin{array}{l}\tilde{\sigma} \\
\stackrel{-}{0} \\
\stackrel{\sim}{\circ}\end{array}$ & $\begin{array}{l}\overrightarrow{0} \\
0 \\
\dot{+} \\
\dot{+}\end{array}$ & $\begin{array}{l}\text { H' } \\
\hat{0} \\
\stackrel{0}{0}\end{array}$ & $\begin{array}{l}\overrightarrow{0} \\
\text { ָ̦ } \\
-\end{array}$ & 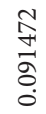 & 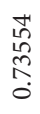 & $\begin{array}{l}+ \\
\infty \\
m \\
n \\
n \\
0\end{array}$ \\
\hline & క్ & $\begin{array}{l}\vec{\infty} \\
\text { ஸे } \\
\stackrel{0}{=} \\
=\end{array}$ & 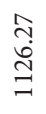 & $\begin{array}{l}\stackrel{0}{N} \\
\tilde{n} \\
\stackrel{i}{n}\end{array}$ & 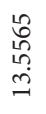 & 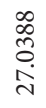 & $\begin{array}{l}\stackrel{n}{0} \\
\stackrel{\infty}{\infty} \\
\dot{m}\end{array}$ & 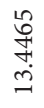 & $\begin{array}{l}1 \\
0 \\
\infty \\
\infty \\
\infty \\
0 \\
0\end{array}$ & 令 & 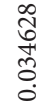 & $\begin{array}{l}\stackrel{\infty}{\mathbb{J}} \\
\stackrel{\infty}{0} \\
\stackrel{0}{0}\end{array}$ & $\begin{array}{l}\text { U. } \\
\infty \\
\infty \\
\infty \\
\text { in }\end{array}$ \\
\hline & ฮี & 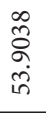 & $\begin{array}{l}\stackrel{0}{\stackrel{N}{N}} \\
\stackrel{i}{0}\end{array}$ & 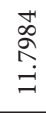 & 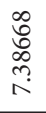 & $\begin{array}{l}\text { f } \\
\text { Oे } \\
\stackrel{-}{二}\end{array}$ & 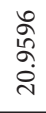 & 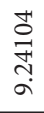 & $\begin{array}{l}\infty \\
\hat{2} \\
\hat{\kappa} \\
\text { in }\end{array}$ & $\underset{\substack{n \\
\stackrel{\infty}{+}}}{\stackrel{\infty}{i}}$ & $\begin{array}{l}8 \\
8 \\
0 \\
0 \\
0\end{array}$ & 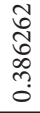 & $\begin{array}{l}\stackrel{+}{N} \\
\text { ळे }\end{array}$ \\
\hline & हี & 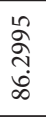 & 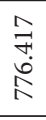 & $\begin{array}{l}m \\
\vec{n} \\
\infty \\
\stackrel{a}{a}\end{array}$ & $\begin{array}{l}\mathscr{N} \\
\infty \\
\stackrel{f}{=} \\
=\end{array}$ & $\begin{array}{l}\hat{N} \\
\text { م. } \\
\text { فें }\end{array}$ & $\begin{array}{l}\stackrel{0}{0} \\
\stackrel{1}{0} \\
\dot{n}\end{array}$ & $\begin{array}{l}\stackrel{10}{\infty} \\
\stackrel{0}{0} \\
\stackrel{0}{0}\end{array}$ & 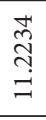 & $\begin{array}{l}\text { ָे } \\
\text { ָे } \\
\text { ì }\end{array}$ & $\begin{array}{l} \\
8 \\
0 \\
0 \\
0 \\
\end{array}$ & 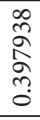 & 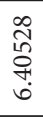 \\
\hline 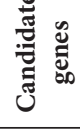 & 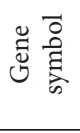 & Oิ & 苻 & ิิ & 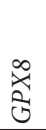 & 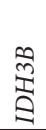 & 安 & స్టె & 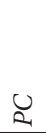 & Оิ & 命 & $\frac{D}{\omega}$ & 命 \\
\hline
\end{tabular}




\begin{tabular}{|c|c|c|c|c|c|c|c|c|c|c|c|c|c|c|}
\hline \multirow{6}{*}{ 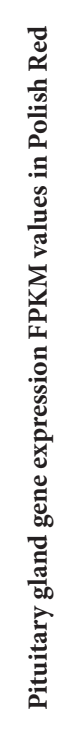 } & 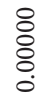 & 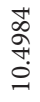 & 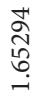 & $\begin{array}{l}\overrightarrow{3} \\
\hat{n} \\
\stackrel{n}{0} \\
\infty\end{array}$ & $\begin{array}{l}\hat{N} \\
\hat{\widehat{O}} \\
\text { ర. }\end{array}$ & $\begin{array}{l}8 \\
8 \\
0 \\
0\end{array}$ & 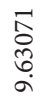 & $\begin{array}{l}0 \\
\infty \\
\infty \\
0 \\
0 \\
\qquad\end{array}$ & 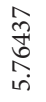 & $\begin{array}{l}\stackrel{2}{2} \\
\hat{\alpha} \\
\text { م. } \\
\infty\end{array}$ & 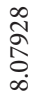 & 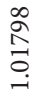 & $\begin{array}{l}\stackrel{H}{\widehat{N}} \\
\stackrel{+}{+}\end{array}$ & 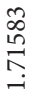 \\
\hline & $\begin{array}{l}8 \\
8 \\
8 \\
0\end{array}$ & $\begin{array}{l}\not \\
\infty \\
\infty \\
\infty \\
\infty \\
0 \\
0\end{array}$ & 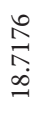 & 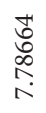 & $\begin{array}{l}\stackrel{\infty}{\%} \\
\text { ڤે } \\
\text { ஸे }\end{array}$ & $\begin{array}{l}8 \\
\vdots \\
\vdots \\
\vdots\end{array}$ & 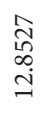 & 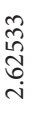 & $\underset{\substack{\hat{N} \\
\text { in } \\
\text { in }}}{ }$ & $\begin{array}{l}\text { ते } \\
\text { ஸे } \\
\dot{r}\end{array}$ & 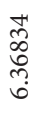 & 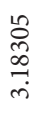 & $\begin{array}{l}\stackrel{0}{N} \\
\hat{\imath} \\
\stackrel{0}{i} \\
i\end{array}$ & 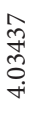 \\
\hline & $\begin{array}{l}8 \\
8 \\
8 \\
0\end{array}$ & $\frac{\stackrel{\circ}{\stackrel{n}{n}}}{\vec{\sigma}}$ & $\begin{array}{l}\overrightarrow{\widehat{a}} \\
\text { ஸे } \\
\text { in }\end{array}$ & 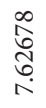 & 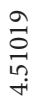 & $\begin{array}{l}\stackrel{8}{0} \\
8 \\
0\end{array}$ & 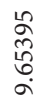 & $\begin{array}{l}\frac{10}{a n} \\
\hat{n} \\
\\
n\end{array}$ & 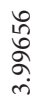 & $\begin{array}{l}\hat{\Omega} \\
\hat{n} \\
\stackrel{n}{n}\end{array}$ & 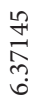 & $\begin{array}{l}\underset{J}{\exists} \\
\vec{i}\end{array}$ & $\begin{array}{l}\text { त̃ } \\
\hat{\hat{D}} \\
\text { in } \\
\text { in }\end{array}$ & 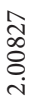 \\
\hline & $\begin{array}{l}8 \\
8 \\
8 \\
0\end{array}$ & $\begin{array}{l}\text { No } \\
\stackrel{\circ}{n} \\
\hat{\sigma}\end{array}$ & 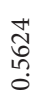 & $\begin{array}{l}\text { 量 } \\
\exists \\
\exists\end{array}$ & 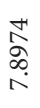 & 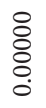 & 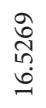 & 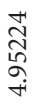 & 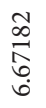 & 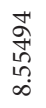 & 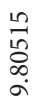 & $\begin{array}{l}0 \\
\infty \\
0 \\
\infty \\
\infty \\
i \\
i\end{array}$ & $\begin{array}{l}\overrightarrow{\vec{m}} \\
\vec{m} \\
\dot{m}\end{array}$ & 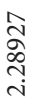 \\
\hline & 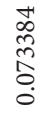 & $\begin{array}{l}\vec{b} \\
\stackrel{1}{n} \\
\infty \\
\infty\end{array}$ & $\begin{array}{l}\stackrel{\infty}{\circ} \\
\stackrel{\vec{\delta}}{+} \\
+\end{array}$ & $\begin{array}{l}2 \\
\infty \\
\infty \\
\infty \\
\pm \\
-1\end{array}$ & $\begin{array}{l}\bar{\sigma} \\
\dot{0} \\
\stackrel{0}{0} \\
\stackrel{0}{0}\end{array}$ & $\begin{array}{l}8 \\
8 \\
0 \\
0 \\
0\end{array}$ & 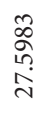 & 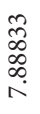 & 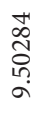 & $\begin{array}{l}\stackrel{H}{ } \\
\text { Oे } \\
\text { తి }\end{array}$ & 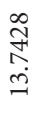 & 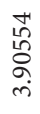 & 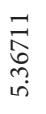 & 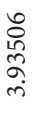 \\
\hline & $\begin{array}{l}8 \\
8 \\
8 \\
0\end{array}$ & 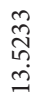 & $\begin{array}{l}\hat{N} \\
\hat{\hat{O}} \\
\stackrel{0}{0} \\
\text { r. }\end{array}$ & 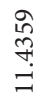 & $\begin{array}{l}\text { ते } \\
\delta \\
\infty \\
1 \\
\infty\end{array}$ & $\begin{array}{l}8 \\
8 \\
8 \\
0\end{array}$ & $\begin{array}{l}0 \\
0 \\
0 \\
0 \\
0\end{array}$ & 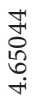 & $\begin{array}{l}0 \\
\infty \\
\tilde{0} \\
0 \\
0 \\
0\end{array}$ & 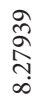 & 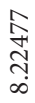 & $\begin{array}{l}\hat{D} \\
\overrightarrow{0} \\
\tilde{m} \\
\ddot{n}\end{array}$ & 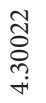 & 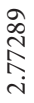 \\
\hline $\begin{array}{l}\text { 岂 } \\
\text { 蛋 }\end{array}$ & $\begin{array}{l}8 \\
8 \\
8 \\
0\end{array}$ & 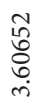 & 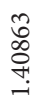 & 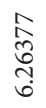 & $\begin{array}{l}\vec{n} \\
\infty \\
\stackrel{\infty}{\hat{n}} \\
\stackrel{n}{n}\end{array}$ & $\begin{array}{l}8 \\
\vdots \\
\vdots \\
0\end{array}$ & 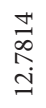 & 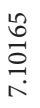 & $\begin{array}{l}10 \\
\hat{2} \\
\swarrow \\
0 \\
0 \\
0\end{array}$ & 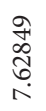 & $\begin{array}{l}\vec{b} \\
\infty \\
\infty \\
\infty \\
\infty\end{array}$ & 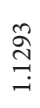 & $\begin{array}{l}\text { Oे } \\
\stackrel{\text { ते }}{i}\end{array}$ & 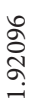 \\
\hline 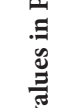 & $\begin{array}{l}\hat{0} \\
\text { N̦} \\
0\end{array}$ & 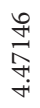 & 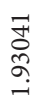 & 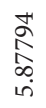 & $\begin{array}{l}H \\
0 \\
0 \\
0 \\
\infty \\
\infty\end{array}$ & \begin{tabular}{l}
$\stackrel{8}{0}$ \\
$\vdots$ \\
\hdashline
\end{tabular} & $\begin{array}{l}\text { Jै } \\
\stackrel{+}{+} \\
\text { in }\end{array}$ & 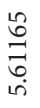 & $\begin{array}{l}\overrightarrow{\vec{b}} \\
\overrightarrow{7} \\
i n\end{array}$ & $\begin{array}{l}\overrightarrow{\vec{N}} \\
\stackrel{\hat{O}}{N}\end{array}$ & 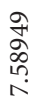 & $\begin{array}{l}\stackrel{2}{\circ} \\
\stackrel{2}{\leftrightarrows}\end{array}$ & 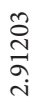 & 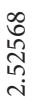 \\
\hline 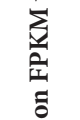 & 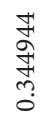 & $\begin{array}{l}\text { 范 } \\
\text { in } \\
\text { in }\end{array}$ & 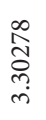 & $\begin{array}{l}\stackrel{10}{0} \\
\stackrel{\infty}{+} \\
\underset{\infty}{\infty}\end{array}$ & $\begin{array}{l}\text { 辛 } \\
\text { †े } \\
\text { in }\end{array}$ & \begin{tabular}{l}
8 \\
$\varnothing$ \\
$\vdots$ \\
\hdashline
\end{tabular} & 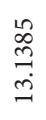 & 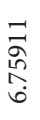 & \begin{tabular}{l} 
Tै \\
\multirow{d}{*}{} \\
$\stackrel{+}{+}$
\end{tabular} & $\begin{array}{l}m \\
\stackrel{m}{H} \\
\vec{b}\end{array}$ & $\begin{array}{l}\hat{n} \\
\hat{N} \\
\hat{n} \\
\sigma\end{array}$ & 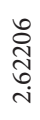 & 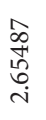 & 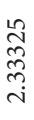 \\
\hline 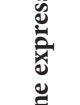 & $\begin{array}{l}8 \\
8 \\
8 \\
0\end{array}$ & $\begin{array}{l}\infty \\
\infty \\
\infty \\
m \\
m\end{array}$ & $\begin{array}{l}\overrightarrow{3} \\
\infty \\
\hat{0} \\
\text { in }\end{array}$ & 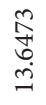 & 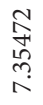 & 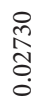 & 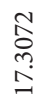 & 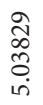 & $\begin{array}{l}\hat{N} \\
\hat{~} \\
\text { જ. }\end{array}$ & 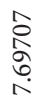 & 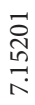 & 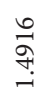 & $\begin{array}{l}\stackrel{0}{\overrightarrow{7}} \\
\underset{\infty}{\infty} \\
\dot{r}\end{array}$ & 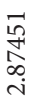 \\
\hline 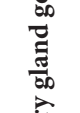 & $\begin{array}{l}\text { m } \\
\text { ஸे } \\
\text { ᄋे } \\
\dot{0}\end{array}$ & 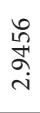 & 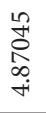 & $\begin{array}{l}\tilde{N} \\
\hat{n} \\
m \\
\infty \\
\infty\end{array}$ & 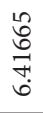 & $\begin{array}{l}8 \\
8 \\
8 \\
0\end{array}$ & $\begin{array}{l}\stackrel{n}{O} \\
\stackrel{-}{2}\end{array}$ & $\begin{array}{l}\stackrel{\infty}{\pi} \\
\stackrel{\alpha}{\sigma} \\
\dot{+}\end{array}$ & $\begin{array}{l}\underset{7}{J} \\
\stackrel{0}{0} \\
\text { in }\end{array}$ & 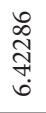 & $\begin{array}{l}\stackrel{0}{0} \\
\infty \\
0 \\
\infty \\
\end{array}$ & 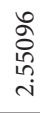 & 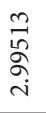 & 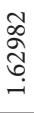 \\
\hline 莺 & $\begin{array}{l}2 \\
0 \\
\sigma \\
\stackrel{2}{0} \\
0\end{array}$ & $\begin{array}{l}\text { gे } \\
\text { †े } \\
\text { o } \\
\text { i }\end{array}$ & $\begin{array}{l}\tilde{N} \\
\tilde{m} \\
\tilde{m} \\
\infty \\
\infty\end{array}$ & $\begin{array}{l}\widetilde{\sigma} \\
\stackrel{2}{2} \\
\stackrel{\infty}{\infty}\end{array}$ & 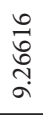 & $\begin{array}{l}8 \\
8 \\
\circ \\
0 \\
0\end{array}$ & $\begin{array}{l}\underset{\infty}{\infty} \\
\stackrel{\infty}{\infty}\end{array}$ & $\begin{array}{l}\stackrel{10}{7} \\
\stackrel{0}{0} \\
\stackrel{+}{+}\end{array}$ & 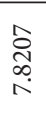 & $\begin{array}{l}\hat{i n} \\
\vec{b} \\
0\end{array}$ & $\begin{array}{l}\frac{0}{2} \\
\frac{1}{0} \\
a\end{array}$ & 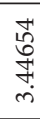 & $\begin{array}{l}3 \\
0 \\
0 \\
\pi \\
\pi \\
\end{array}$ & 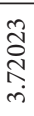 \\
\hline 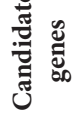 & 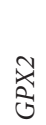 & 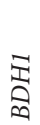 & $\sum_{i 1}$ & Uु & $I$ & 苍 & 疍 & $\pi$ & $\underset{⿱ 乛 龰}{\mathbb{Z}}$ & 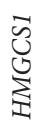 & 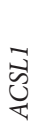 & $\overrightarrow{\mathbb{N}}$ & 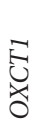 & 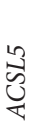 \\
\hline
\end{tabular}


as moderately expressed (FPKM values: $>10$ to $<40$ ), and averagely expressed SOD3, GPX5, GPX6, GPX7, GPX2, GPX8, BDH1, ACSL3, ACSL6, ACSL4, CPT1A, and OXCT1 respectively, in Polish HF and Polish Red breeds. In case of pituitary gland gene expression profiling, the SOD1 and $G P x 3$ were highly expressed (FPKM values of $>40$ ), followed by SOD2, GPX8, IDH3B, ACAT1, ACSL4, and PC as moderately expressed (FPKM values: $>10$ to $<40$ ), and averagely expressed SOD3, GPX3,GPX5, GPX6, GPX7, GPX2, BDH1, BDH2, ACSL3, ACSL6, CPT1A, OXCT1, FN1, HMGCL, HMGCS2, ACAT2, ACAT1, HMGCS1, ACSL1 and ACSL5 respectively, in Polish HF and Polish Red breeds.

\section{Conclusions}

Understanding the genetic factors that predispose metabolic disorders in cattle would benefit the dairy industry by providing producers, breeding services, and veterinary practitioners a tool to forecast a cow's susceptibility to metabolic disorders. Considering presented results on hepatic and pituitary gland gene expression profiling study, a further research is an essential pre-requisite to validate the identified candidate genes.

\section{Acknowledgement}

This research paper was financially supported by National Science Centre, Krakow, Poland, Project No. 2012/05/B/NZ2/01629; entitled "Analiza transkryptomów genomu Bos taurus przy zastosowaniu technologii sekwencjonowania kolejnej generacji”.

\section{References}


1. Kroezen V, Schenkel FS, Miglior F, Baes CF, Squires EJ. Candidate gene association analyses for ketosis resistance in Holsteins. J Dairy Sci. 2018;101:52405249.

2. Biswal S, Nayak DC, Sardar KK Prevalence of ketosis in dairy cows in milk shed areas of Odisha state, India. Vet World. 2016;9:1242-1247.

3. Raboisson D., Mounié M., Khenifard E., Maigné E The economic impact of subclinical ketosis at the farm level: Tacklingthe challenge of over-estimation due to multiple interactions. Prev Vet Med. 2015;122: 417-425.

4. Herdt T.H. Ruminant adaptation to negative energy balance. Influences on the etiology of ketosis and fatty liver. Vet Clin North Am Food Anim Pract. 2000;16:215-230.

5. Bernabucci U., Ronchi B., Lacetera N., Nardone A.: Influence of Body Condition Score on Relationships Between Metabolic Status and Oxidative Stress in Periparturient Dairy Cows. J Dairy Sci. 2005;88:2017-2026.

6. Sordillo L.M., Aitken S.L Impact of oxidative stress on the health and immune function of dairy cattle. Vet Immunol Immunop. 2009;128:104-109.

7. Raboisson D., Mounié M., Maigné E. Diseases, reproductive performance, and changes in milk production associated with subclinical ketosis in dairy cows: A meta-analysis and review. J Dairy Sci. 2014;97:7547-7563.

8. Contreras GA, O’Boyle NJ, Herdt TH, et al. Lipomobilization in periparturient dairy cows influences the composition of plasma nonesterified fatty acids and leukocyte phospholipid fatty acids. J Dairy Sci. 2010;93:2508-2516.

9. Dechow C.D, Rogers G.W, Sander-Nielsen U., et al.: Correlations among body condition scores from various sources, dairy form, and cow health from the United States and Denmark. J Dairy Sci. 2004;87:3526-3533.

10. Janovick-Guretzky N. A., Dann H. M., Carlson D. B. , Murphy M. R., Loor J. J., Drackley J. K.: Housekeeping Gene Expression in Bovine Liver is Affected by Physiological State, Feed Intake, and Dietary Treatment J Dairy Sci. 2007;90:2246-2252.

11. Shi X., Li D., Deng Q., Peng Z., Zhao C., Li X., Wang Z., Li X., Liu G. Acetoacetic acid induces oxidative stress to inhibit the assembly of very low density lipoprotein in bovine hepatocytes J Dairy Res. 2016;83:442-446.

12. Berge A.C., Vertenten G. A field study to determine the prevalence, dairy herd management systems, and fresh cow clinical conditions associated with ketosis in western European dairy herds. J Dairy Sci. 2014;97:2145-2154 
13. Pryce J. E., Parker Gaddis K. L., Koeck A. et al. Invited review: Opportunities for genetic improvement of metabolic diseases. J Dairy Sci. 2016;99:68556873.

14. Pareek CS, Błaszczyk P, Dziuba P, Czarnik U, Fraser L, Sobiech P, et al. Single nucleotide polymorphism discovery in bovine liver using RNA-seq technology. PLoS ONE 2017;12:e0172687.

15. van Dorland, H.A., Graber, M., Kohler, S., Steiner, A., Bruckmaier, R.M. Comparison of hepatic adaptation in extreme metabolic phenotypes observed in early lactation dairy cows on-farm. J Anim Physio Anim Nut. 2013;98:693703.

16. van Dorland, H.A., Richter, S., Morel, I., Doherr, M.G., Castro, N., Bruckmaier, R.M. Variation in hepatic regulation of metabolism during the dry period and in early lactation in dairy cows. . J Dairy Sci. 2009;92:1924-1940.

17. Cánovas, A., Rincón, G., Islas-Trejo, A., Jimenez-Flores, R., Laubscher, A., Medrano, F.. RNA sequencing to study gene expression and single nucleotide polymorphism variation associated with citrate content in cow milk. . J Dairy Sci. 2013;96:2637- 2648.

18. Khan, M.J., Hosseini, A., Burrell, S., Rocco, S.M., McNamara, J.P., Loor, J.J. Change in subcutaneous adipose tissue metabolism and gene network expression during the transition period in dairy cows, including differences due to sire genetic merit. . J Dairy Sci. 2013;96:2171-2182.

19. Weber, C., Hametner, C., Tuchscherer, A., Losand, B., Kanitz, E., Otten, W., Sauerwein, H., Bruckmaier, R.M., Becker, F., Kanitz, W., Hammon, H.M.. Hepatic gene expression involved in glucose and lipid metabolism in transition cows: Effects of fat mobilization during early lactation in relation to milk performance and metabolic changes. . J Dairy Sci. 2013a;96:5670-5681.

20. Weber, C., Hametner, C., Tuchscherer, A., Losand, B., Kanitz, E., Otten, W., Singh, S.P., Bruckmaier, R.M., Becker, F., Kanitz, W., Hammon, H.M. Variation in fat mobilization during early lactation differently affects feed intake, body condition, and lipid and glucose metabolism in high-yielding dairy cows. . J Dairy Sci. 2013b;96:165-180.

21. Clempson, A.M., Pollott, G.E., Brickell, J.S., Wathes, D.C. Associations between bovine IGFBP2 polymorphisms with fertility, milk production, and metabolic status in UK dairy cows. Anim Biotech. 2012;23:101-113.

22. Tetens, J., Seidenspinner, T., Buttchereit, N., Thaller, G. Whole-genome association study for energy balance and fat/protein ratio in German Holstein bull dams. Anim Genet. 2012;44:1-8. 
23. Buitenhuis, A.J., Sundekilde, U.K., Poulsen, N.A., Bertram, H.C., Larsen, L.B., Sorensen, P. Estimation of genetic parameters and detection of quantitative trait loci for metabolites in Danish Holstein milk. J Dairy Sci. 2013;96:32853295.

24. Parker Gaddis, K.L., Cole, J.B., Clay, J.S., Maltecca, C. Genomic selection for producer-recorded health event data in US dairy cattle. J Dairy Sci. 2014;97:3190-3199.

25. Parker Gaddis K.L., Megonigal J.H., Clay J.S., Wolfe C.W. Genome-wide association study for ketosis in US Jerseys using producer-recorded data. . J Dairy Sci. 2018;101:413-424.

26. Mahmoudi, A., Zargaran, A., Amini, H.R., Assadi, A., Hokmabad, R.V., Eghbalsaied, S. A SNP in the 3'-untranslated region of AMPK $\gamma 1$ may associate with serum ketone body production of Holstein dairy cows. Gene. 2015;574:48-52.

27. Tetens, J., Heuer, C., Heyer, I., Klein, M.S., Gronwald, W., Junge, W., Oefner, P.J., Thaller, G., Krattenmacher, N. Polymorphisms within the APOBR gene are highly associated with milk levels of prognostic ketosis biomarkers in dairy cows. Physio Genom. 2015;47:129-137.

28. Chomczynski P, Sacchi N. Single-step method of RNA isolation by acid guanidinium thiocyanate-phenol-chloroform extraction. Anal Biochem. 1987;162:156-159.

29. Martin MC. Cutadapt removes adapter sequences from high-throughput sequencing reads. EMBnetjournal, 2011. North America.17 (http://journal. embnet.org/index.php/embnetjournal/article/view/200/479).

30. Li H, Durbin R. Fast and accurate short read alignment with Burrows-Wheeler transform. Bioinformatics. 2009;25:1754-1760.

31. Anders S, Pyl PT, Huber W. HTSeq-a Python framework to work with high-throughput sequencing data. Bioinformatics. 2015;1:166-169.

32. Love MI, Huber W, Anders S. Moderated estimation of fold change and dispersion for RNA-seq data with DESeq2. Genome Biol. 2014;15:550. 